\title{
Research and Application of Smart Hanger Based on Airbag Inflation Model and Multi-object Collaborative Optimization Algorithm
}

\author{
Yihong Zhang ${ }^{1,2, a}$, Xi Jin ${ }^{1, b}$ and Zhijie Wang ${ }^{1,2, c}$ \\ ${ }^{1}$ College of Information Science \&Technology, DongHua University, Shanghai 201620, China; \\ ${ }^{2}$ Engineering Research Center of Digitized Textile and Fashion Technology, Ministry of Education \\ China; \\ azhangyh@dhu.edu.cn, bkimhe8@126.com, czjwang@dhu.edu.cn
}

Keywords: Smart Hanger, Garment defect detecting, Flexible pressure sensor, Adaptive fuzzy control, Airbag inflation model, Multi objective optimization.

\begin{abstract}
This paper presents a smart hanger based on airbag inflation and muti-object collaborative optimization algorithm for intelligent garment inspecting system. To fully extend the garment to a proper position, one fuzzy Self-adaptive PID method is given to control the pneumatic motor by comparing the feedback value of the flexible pressure sensor with the threshold value. Flexible sensor arrays were embedded on the surface of the smart hanger; the pressure value detected shows appropriate degree between cloths and the smart hanger, and it's an important standard for garment inspection. One multi-objective optimization algorithm is proposed for the various targets control on the smart hanger in order to achieve the optimal value.
\end{abstract}

\section{Introduction}

An smart hanger model[1] based on graphite alkenes sensor and airbag inflation is presented in this paper, ths smart hanger is applied for the garment defect detecting research area. Figure 1 illustrated the working theory diagram of the smart hanger.

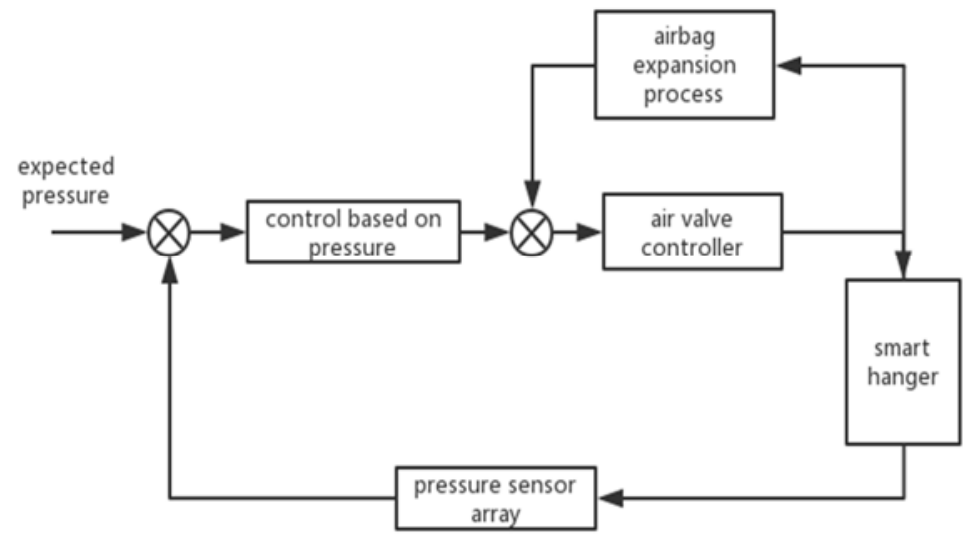

Fig. 1 Working theory diagram of the smart hanger

The clothing pressure [2][3] refers to the pressure caused by limits of clothing, garment shape, size, weight and other factors to the human body, or when loose amount of clothing is not adapted to the slipping amount of skin due to the stretching of the skin and needs the elastic extension of clothes to compensate. It is a factor which cannot be ignored in the garment defect detecting [4][5] research area for image capturing[6]. In this paper, The pressure measurement of different parts needs to take advantage of sensor array consisting of multiple sensors, and the materials are flexible graphite alkenes pressure sensors[7] and airbag inflations[8][9]. The flexible graphite alkenes pressure sensors are equipped with characteristics like flexibility, high sensitivity and so on, which made it very important for this paper. Fig.2 illustrated the structure of airbag inflations and effective pressure value 
simulation results. The effective value part on the arm part of the smart hanger is illustrated by using bule color.

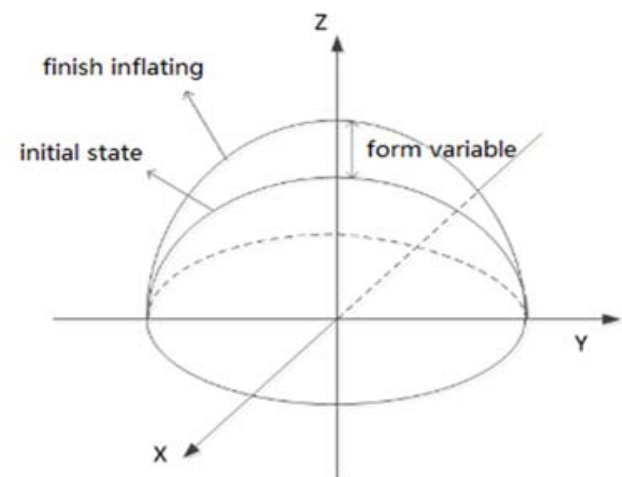

a

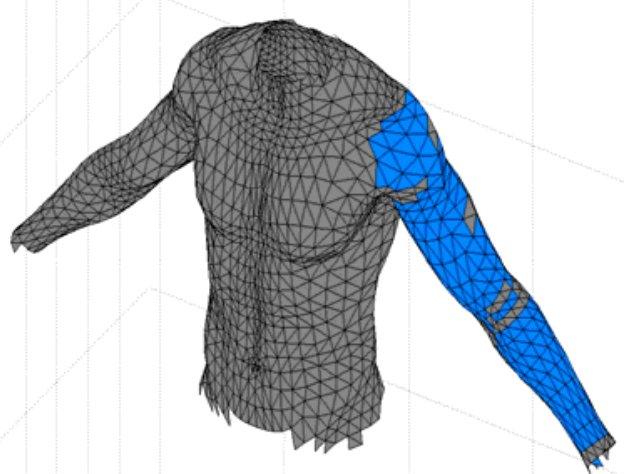

b

Fig.2 a. The structure of airbag inflations and b. The effective pressure value simulation results .

When establishing the mathematical model of air pressure sensors, an experimental modeling method was conducted. The process of building up arm models was listed in this paper. With the change of test location, the mathematical model of airbag would change accordingly and needed to be changed in the controlling process.
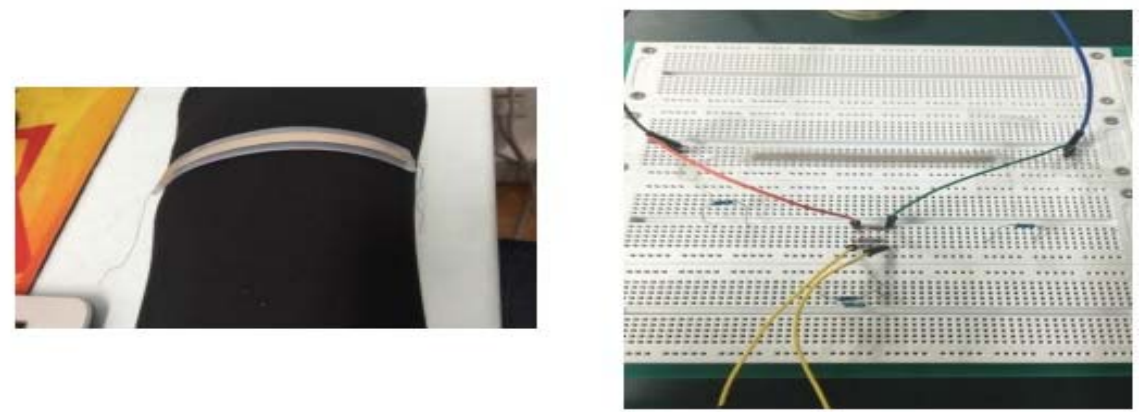

Fig. 3 The pressure value obtaining and testing experiment

The dynamic model of intelligent hanger and the parameters' self-tuning fuzzy control strategy was conducted. And then the plane measurement in stationary state and the curved surface measurement experiment would be conducted. Figure 3 illustrated the pressure value obtaining and testing experiment.And different trends and results were accompanied by plane and curved surface measurement under different conditions of materials with different quality, so it is necessary to conduct a weight analysis on multiple target functions to get material parameter that meets as much targets as possible. Multi-object collaborative optimization algorithm [14][15] is also proposed in this paper to achieve the optimal value relative for the different targets on the smart hanger.

\section{Self-tuning fuzzy Control Simulation}

The parameter self-adaptive fuzzy control program was written in MATLAB according to the dynamic model. Its flow diagram of parameter self-adaptive fuzzy control is illustrated in Figure 4. Acoording to the charracter of the smart hanger, self-adaptive fuzzy control object is defined as equation (1):

$$
\begin{aligned}
\Delta u(k) & =K_{p}[e(k)-e(k-1)]+K_{I} e(k)+K_{D}[e(k)-2 e(k-1)+e(k-2)] \\
& =K_{p} e_{c}(k)+K_{I} e(k)+K_{D}\left[e_{c}(k)-e_{c}(k-1)\right]
\end{aligned}
$$

And it is incremental pid, In the formula(1), e means deviation of force, ec means diviation change rate of force . Kp,ki,kd are fuzzy logic tuing PID parameters with proper fuzzy rules. Formulate the fuzzy inference rules based on membership function that are formulated by input and output. The rules descript in following form: if $(\mathrm{e}$ is $*)$ and $(\mathrm{ec}$ is $*)$ then $(\mathrm{kp}$ is $*)(\mathrm{ki}$ is $*)(\mathrm{kd} \mathrm{is} *)$.Table 1 illustrates the inuput and output parameter of discourse domain. Table 1 illustrated the input and output of discourse domain. 
Table 1. Input and output of discourse domain

\begin{tabular}{|c|c|}
\hline parameter & $\begin{array}{c}\text { discourse } \\
\text { domain }\end{array}$ \\
\hline $\mathrm{e}$ & {$[-0.3,0.3]$} \\
\hline$e_{c}$ & {$[-30,30]$} \\
\hline$\Delta k_{P}$ & {$[-0.2,0.2]$} \\
\hline$\Delta k_{I}$ & {$[-0.1,0.1]$} \\
\hline$\Delta k_{D}$ & {$[-3 \mathrm{e}-4,3 \mathrm{e}-4]$} \\
\hline
\end{tabular}

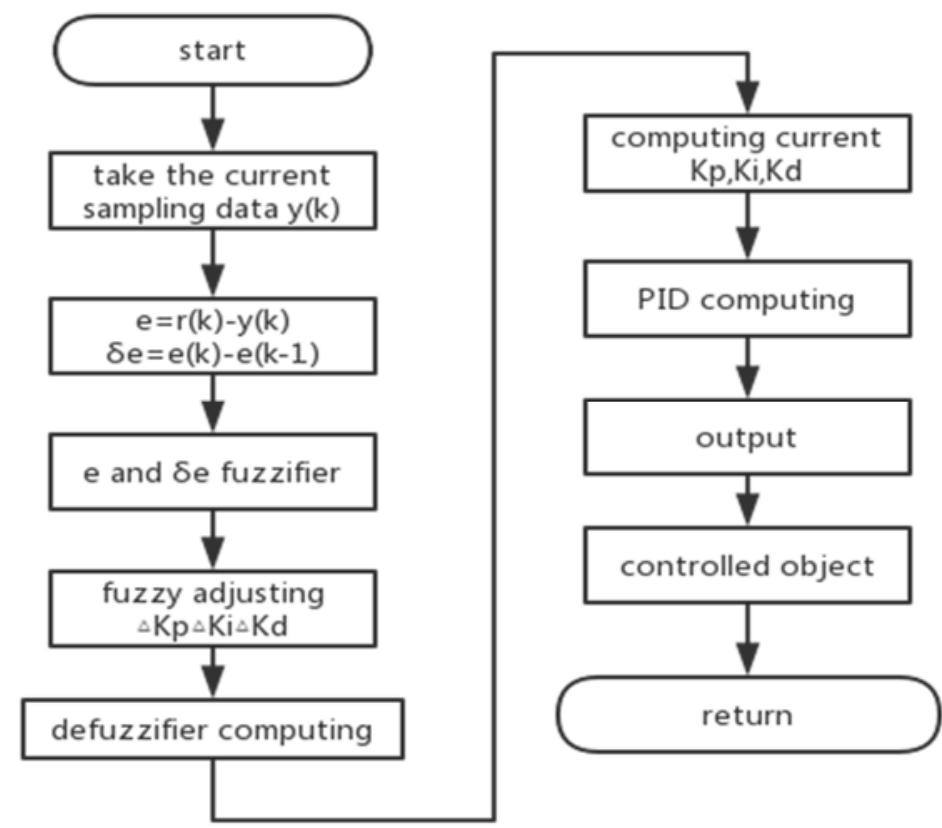

Fig. 4 The flow diagram of parameter self-adaptive fuzzy control

\section{Simulation and Experimental Research on Non-Sensor Noise Control}

Balloon cyst pressure sensor array in this article controls changes for air bags when servo motors inflate and deflate the air bags, and reflect the fit of clothing by making use of changes clothing pressure between clothes and air bags when air bags change.

In control process, rise time $t_{r}$ presents the time required for reaching stable value for first time, peak time $t_{p}$ stands for the time the response curve needs to rise from 0 to the first peak, and maximum overshot refers to the difference between the maximum peak and steady-state value of response curve, generally written as percentages. The overshoot is only connected with damping ratio, and setting time $t_{s}$ refers to the time response curve needs to arrive and break within allowed error ranges (steadystate worth equals to $\pm 2 \%$ or $\pm 5 \%$, and $\pm 5 \%$ is used in the paper). Comparing the traditional PID and fuzzy control PID, it can be found that after parameter self-tuning control, difference between rise time is not very large, while peak time, overshoot and time for adjustment reduce significantly, which reflects the superiority of fuzzy control.

The measured parts in the paper include shoulder, chest and waist. Because sizes of different human parts are different, the shapes of hanger in these three parts are not the same. So before the control process, models of these three a parts are different. In this paper, the enlargement process for balloons is simulated in the experiment and the model was built. The same method was used to analyze and later correspondingly control parts beyond the shoulder, and differences can be seen from simulation process. Coupled with the different responses of different parts for clothing pressure, when 
discussing the comfort of clothing, the appropriate changes must be taken, such as steady-state value in this article of $0.3 \mathrm{~N}$ on the shoulder and waist $0.9 \mathrm{~N}$. Figures 5 and Figure 6 showed that the time of stability of fuzzy PID controlling process were 4.8 seconds and 15 seconds.

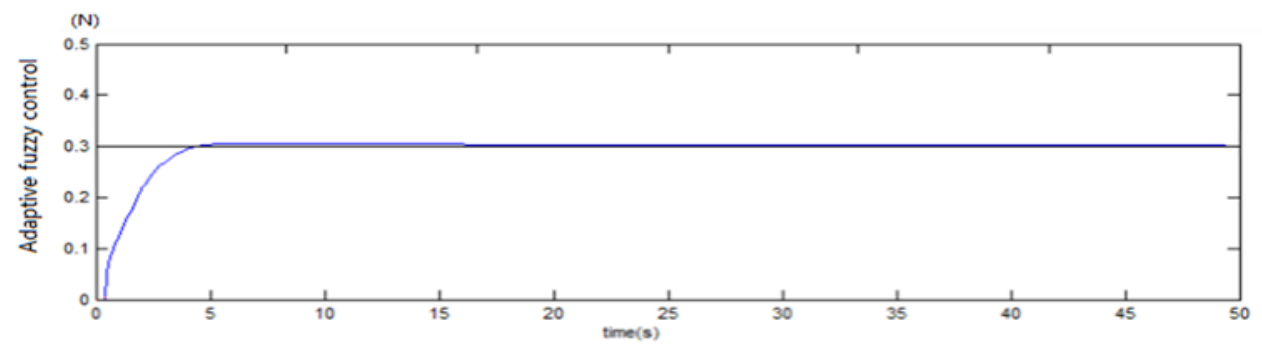

Fig.5 Pressure control process on shoulders

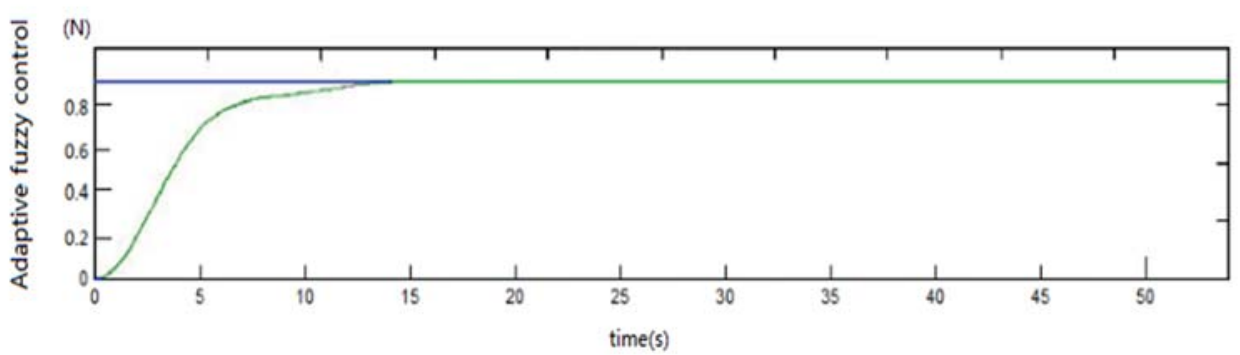

Fig.6 Pressure control process on waists

\section{Simulation of Multi-Object Collaborative Optimization}

Multi-object collaborative optimization model based on ant colony optimization algorithm [16][17] is proposed in this paper. Parameter $\mathrm{f}$ is defined as optimization target in Equation (2), $\mathrm{p}$ is drift, $\mathrm{c}$ is repeatability, 1 is linearity, e is defined as hysteresis and $\mathrm{r}$ is surface test, and all parameters are based on the parameters recommended by sensor companies, so the constraint conditions of these 5 objectives is defined as Equation (3).

$$
\begin{aligned}
& \mathrm{f}=\frac{c^{2}}{e^{2}+p^{2}+l^{2}} \\
& \text { St: }\left\{\begin{aligned}
-0.05 & <p<0.05 \\
0 & <c<8 \\
0.03 & <l<0.01 \\
3 & <e<10 \\
8 & <r<51.7
\end{aligned}\right.
\end{aligned}
$$

Because the different clothes have different features, and with different materials, the requirements for comfort of clothes when wearing them are different. The turnouts need to be breathable and convenient for action, and their appearance materials can protect people from harm. So the pressure of fire clothing will concentrate in shoulders when wearing them, mainly from the weight of clothes. While in terms of waist, chest and arms and other parts, in order to show the ventilation and lightness, there cannot be too much pressure. The thick coat is different form turnouts. Coats need to protect people from wind and to keep warm, which makes clothes fit to the body in the production process and the clothing pressure will be dispersed in various parts.

Intelligent hanger is designed for garments detection to detect the clothing pressure of garments with different materials. Due to differences in clothing materials in a balloon model, the parameters will change accordingly. In order to find out dynamic model and control the process effectively, extensive data collection is required so that dynamic models are more reliable.Figure.7 illustrated the diagram of ant colony optimization algorithm. 


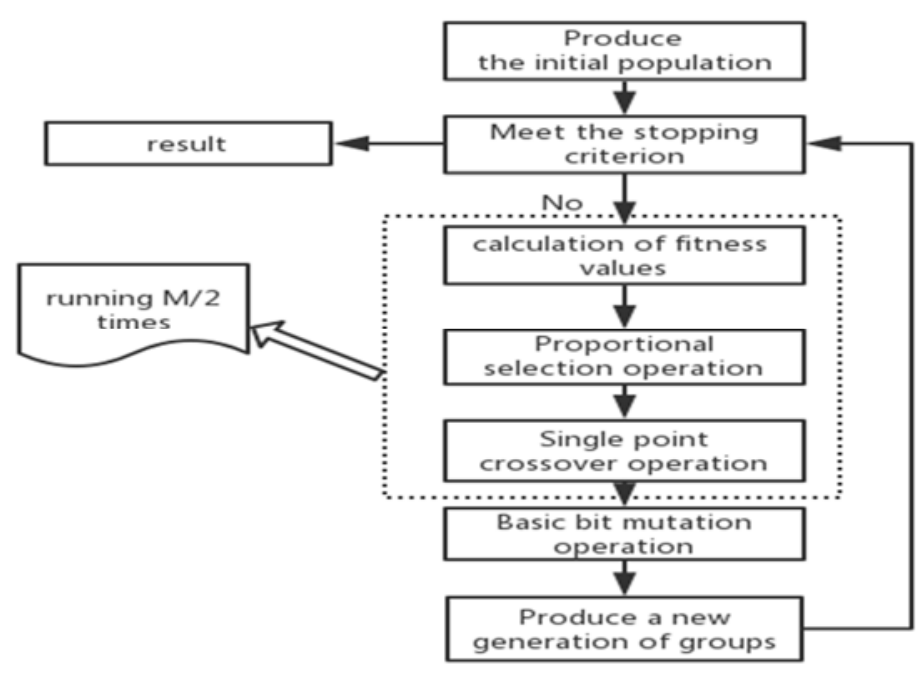

Fig. 7 The diagram of ant colony optimization algorithm.

The adaptability of fuzzy control PID controller was further verified.In sensors of parameters selftuning fuzzy control model above, verifying the garments of different materials can compare a number of different important parameters.Pressure value is set as $30 \mathrm{~g}$ and $20 \mathrm{~g}$, and the actuation duration is respectively $2 \mathrm{~min}$. When placing weights on the sensor, the sensor displays pressure values, and after $3 \mathrm{~min}$, it reaches a steady value. The degrees of stability are $96 \%$ and $93 \%$, and after $10 \mathrm{~min}$, it reaches $98 \%, 97 \%$. The stability has improved significantly over time.30g, 20g and $10 \mathrm{G}$ were typed every $5 \mathrm{~min}$ following a descending order, with each input value repeating 20 times. According to test results, the mean and standard deviation were $25.65 \mathrm{~g} \pm 1.03 \mathrm{~g}, 0.48 \mathrm{~g} \pm 0.86 \mathrm{~g}$ and $7.28 \mathrm{G} \pm 0.48 \mathrm{~g}$ when the input values were $30 \mathrm{~g}, 20 \mathrm{G}$, and $10 \mathrm{G}$ respectively, and coefficient of variation 2.3, 3.2 and 6.6, showing that higher quality brought better repetition performance.

According to the product instructions of graphite alkenes sensor, the fact that drift $<3 /$ logarithmic time, reproducibility $<5$, linearity $<5$ had no use of explanation on the curved surface.Drift tests showed that the output increases over time, and the degree of stability reached 96 and $98 \%$ the input values were $5 \mathrm{~min}$ and $10 \mathrm{~min}$. Although what was provided were continuous real-time outputs, due to the effect of drift and linearity, the output values immediately read after entering the input values were less than those after they become stable. This means that static measurement is the most suitable way, but if the required accuracy is not high, dynamically test can also be conducted.

Repeatability, linearity and hysteresis all need to meet the measurement requirements. Compared with 21.7 lagging of the company' early sensors, the hysteresis witness a considerable improvement. Therefore, plane measurement in static state can be drawn by testing drift, repeatability, linearity and lagging.

\section{Summary}

In this paper, an intelligent hanger based on self-adaptive fuzzy control and multi-objective optimization algorithm was proposed. The parameter self-adaptive fuzzy control expriments was conducted according to the dynamic model. The comparison between models of parameter selfadjusting control of clothes made of different materials can start from drifting, and repeatability, linearity and retardation and other factors. It was found that the different trends and results were accompanied by plane and curved surface measurement under different conditions of materials with different quality. Multi-object collaborative optimization model based on ant colony optimization algorithm proposed in this paper is proved to be applicable and optimal for the smart hanger control for various garments on it. 


\section{Acknowledgements}

This research was financially supported by the ShangHai Science Foundation-"The Research and Application of Intelligent Garment Defect Detecting based on Cloud Computing”, Funding Number: 14ZR1400400.

This research work was supported by the China Central University Foundation.Project Number: $15 \mathrm{D} 110406$.

\section{References}

[1]. Truong D Q, Ahn K K. Force control for hydraulic load simulator using self-tuning grey predictor - fuzzy PID [J]. Mechatronics, 2009, 19(2):233-246.

[2]. Y.Na Clothing pressure and physiological responses according to boning type of non-stretchable corsets [J]. Fibers and Polymers, 2015, 16(2):471-478.

[3]. Galer S, Beeck M O D, Urbain C, et al. Investigating the Neural Correlates of the Stroop Effect with Magnetoencephalography[J]. Brain Topography, 2015, 28(1):95.

[4]. H.İ. Çelik, L.C. Dülger, M. Topalbekiroğlu. Development of a machine vision system: real-time fabric defect detection and classification with neural networks[J]. Journal of the Textile Institute, 2014, 105(6):575-585.

[5]. Lee D, Park S H, Moon S. Utility-based association rule mining: A marketing solution for crossselling [J]. Expert Systems with Applications, 2013, 40(7):2715-2725.

[6]. Kamino T, Tomimatsu T. Method for manufacturing image capturing device and image capturing device [J]. 2017.

[7]. Sun S, Han B, Jiang S, et al. Nano graphite platelets-enabled piezoresistive cementitious composites for structural health monitoring [J]. Construction \& Building Materials, 2017, 136:314-328.

[8]. Rath A L, Jernigan M V, Stitzel J D, et al. The effects of depowered airbags on skin injuries in frontal automobile crashes.[J]. Plastic \& Reconstructive Surgery, 2005, 115(2):428.

[9]. Cheng Han. Simulation study of airbag process [D]. Nanjing University of Aeronautics \& Astronautics, 2009.

[10]. Luo X. Design and Experimental Study of Pre-mill Fuzzy Control System [J]. Metal Mine, 2012.

[11]. Boulkroune A, M'Saad M. On the design of observer-based fuzzy adaptive controller for nonlinear systems with unknown control gain sign[J]. Fuzzy Sets \& Systems, 2012, 201(201):7185.

[12]. Z. Chen, The application of fuzzy PID control strategy in automotive power steering system [J]. electronic technology and software engineering, 2016 (12): 250-250.

[13]. Tawfeek M A, El-Sisi A B, Keshk A E, et al. Virtual Machine Placement Based on Ant Colony Optimization for Minimizing Resource Wastage[C]// International Conference on Advanced Machine Learning Technologies and Applications. Springer International Publishing, 2014:153164.

[14]. Deepalakshmi P, Radhakrishnan S. An ant colony-based multi objective quality of service routing for mobile ad hoc, networks[J]. EURASIP Journal on Wireless Communications and Networking, 2011, 2011(1):1-12.

[15]. P.R. KVarma, V.V.Kumari, S.S. Kumar. Feature Selection Using Relative Fuzzy Entropy and Ant Colony Optimization Applied to Real-time Intrusion Detection System $\_[\mathrm{J}]$. Procedia Computer Science, 2016, 85:503-510.

[16]. Y.Zhou, F.He, Y.Qiu. Dynamic strategy based parallel ant colony optimization on GPUs for TSPs [J]. Science China Information Sciences, 2017, 60(6):068102. 